Dominika Rokicka, Marta Wróbel, Aleksandra Szymborska-Kajanek, Anna Bożek, Krzysztof Strojek

Clinical Department of Internal Medicine, Diabetology and Cardiometabolic Diseases, School of Medicine in Zabrze Medical University of Silesiain Katowice

\title{
Assessment of compliance to self monitoring of blood glucose in type 2 diabetic patients and level of implementation of Polish Diabetes Association Recommendation for general practitioners - results of multicenter, prospective educational health programme - DIABCON study
}

\begin{abstract}
Introduction. Self monitoring of blood glucose is one of basic elements of effective diabetes treatment. Due to proper self monitoring of blood glucose adequate modification of diabetes treatment - fulfilling criteria of good glycemic control - is possible. Effective treatment enables prevention of late diabetic complications - a cause of disability or death in diabetic population. Material and methods. 765 type 2 diabetic patients (above $18 \mathrm{yrs}$ ) treated with both oral hypoglycaemic drugs or insulin in different schemes were observed (mean age: $64.5 \pm 10.9 \mathrm{yrs}$ ). Therapy was in line with general standards of treatment, medication choice was based on indications and previous independent physician decision. Patients were given glucometer Con-
\end{abstract}

Address for correspondence:

dr n. med. Dominika Rokicka

Oddział Kliniczny Chorób Wewnętrznych, Diabetologii

Schorzeń Kardiometabolicznych

Śląskie Centrum Chorób Serca w Zabrzu

ul. Marii Curie-Skłodowskiej 10, 41-800 Zabrze

Phone: + 48608578989

e-mail: dominika.rokicka@poczta.fm

Translation: lek. Jędrzej Toczko

Clinical Diabetology 2018, 7, 3, 129-135

DOI: 10.5603/DK.2018.0008

Received: 24.01.2018

Accepted: 06.02.2018
tourPlus and were taught how to use it and how often measurement had to be taken (on the basis of Polish Diabetes Association Recommendation for 2016). After 3 months ( \pm 2 weeks) assessment of compliance to self monitoring of blood glucose was performed. Additionally, level of implementation of Polish Diabetes Association Recommendation for general practitioners, functionality of glucometer and usefulness of "second chance" measurement were evaluated.

Results. 440 participants completely fulfilled recommendations $(65.1 \%)$ of general practitioner (investigator) regarding self monitoring documentation (type of documentation provided: glucometer, glucose monitoring diary). In investigators opinion, 315 patients (below $50 \%$ ) were conscientiously compliant to self monitoring of blood glucose based on Polish Diabetes Association Recommendations. In general practice for about $68.0 \%$ of patients $(n=506)$ Polish Diabetes Association Recommendations for self blood glucose monitoring were implemented. Based on investigators opinion the most important features of glucometers which determine effectiveness of self monitoring of glucose are: simplicity, high capacity memory, accuracy and precision of measurement, evaluation of mean glucose in general and mean pre-/after meal. $67.7 \%$ patients $(n=451)$ regarded possibility of "second 
chance" measurement in glucometer ContourPlus as useful in daily self monitoring of blood glucose.

Conclusions. Discrepancy between Polish Diabetes Association Recommendations of self blood glucose monitoring and its realization by type 2 diabetic patients, carried out by general practitioners, was observed. Effective glucose monitoring is dependent on tight cooperation between doctor and patient. (Clin Diabetol 2018; 7, 3: 129-135)

Key words: type 2 diabetes, self monitoring of glycaemia

\section{Introduction}

In a world of expanding civilization, diminishing physical activity, harmful dietary habits and an increasing obesity epidemic, diabetes is a constantly growing issue. In the year of 2000, 200 million people were affected by diabetes worldwide. It was then estimated that in 2030, there would have been 366 million diabetic patients [1]. However, by latest estimations, there are currently $\mathbf{4 1 5}$ million diabetic patients in the world. According to $\mathrm{WHO}$ predictions, the diabetic population will constantly grow, and reach 642 million in 2040 [2]. Diabetes is the cause of nearly 1.5 million deaths annually. Rough calculations show that in $\mathbf{2 0 3 0}$ diabetes will be the $7^{\text {th }}$ most common cause of death worldwide. This numbers allow defining diabetes as a social disease and acknowledging it as a pressing concern of modern and future medicine. It must also be remembered that as a result of longer life expectancy in both general and diabetic populations, the costs of treatment will burden healthcare budgets not only because of prevalence, but also due to occurrence of late complications.

Hyperglycaemia is the main factor contributing to development of chronic complications in diabetes. It induces intracellular disorders of the polyol pathway, non-enzymatic glycation of proteins and protein kinase activation, leading to extensive free-radicals synthesis, resulting in exacerbation of the proliferation and inflammatory processes within the vessel wall [3]. If the patient has other cardiovascular risks present, the development of complications - macrovascular complications in particular - is much faster. This is why multimodal therapy - including not only hypoglycaemic, but also hypotensive and hypolipidemic treatment as well as a behavioural approach — is so important. The importance of an intensive multifactorial intervention was demonstrated in the Steno-2 study. The results of the study remain a cornerstone of modern diabetology, as implementation of multimodal therapy allows saving the life of 1 in 8 patients in a 13 year timeframe [4]
Above all, an effective treatment of diabetes is based on early diagnosis of the disease and other cardiovascular risk factors, as well as quick implementation of adequate therapy. The next stages include proper control of glycaemia, blood pressure, body mass and cholesterol levels. Most of these elements should be controlled by the patient himself. Self monitoring of blood glucose plays a vital role in hypoglycaemic treatment. Due to its proper implementation, adequate modification of diabetes treatment - fulfilling criteria of good glycaemic control - is possible. Widespread introduction of glucometers, enabling relatively precise, real time blood glucose levels monitoring, has been revolutionary to everyday care of diabetic patients. Because of the growing incidence of diabetes and its chronic character, general practitioners introduce the shared care model for diabetic patients more frequently. It relies on planned, regular control in a specialist outpatient clinic together with constant monitoring in the basic healthcare setting.

The aim of this study was to assess the compliance to doctors' guidance regarding self monitoring of blood glucose and the level of implementation of Polish Diabetes Association Recommendations for general practitioners. Specific features of glucometers involved were also evaluated by investigators and patients.

\section{Material and methods}

The DIABCON study was a non-interventional, health education programme. Patients over 18 years of age with type 2 diabetes, requiring regular glycaemia self monitoring with a glucometer were included into the study, after signing Informed Consent Agreement and a consent for processing of personal data. A population of 765 patients was observed. 5 patients were included into the study despite not meeting one of the inclusion criteria - type 2 diabetes. These patients' data was excluded from analysis.

Participants were treated in line with general standards of treatment; medication choice was based on indications and previous independent physician decision. Patients visited the Centre following a standard procedure applying to treatment of their underlying disease. During the first visit (V1), they received a Contour Plus ${ }^{\circledR}$ glucometer, dedicated to self monitoring of blood glucose levels at home. The physician instructed the patient on using the Contour Plus ${ }^{\circledR}$ glucometer, including pre- and post-prandial glycaemia measurements and the "second chance" measurement. Depending on the choice of hypoglycaemic medication, the investigator informed the patient on proper frequency of self monitoring measurements and marked his choice in the Patient Observation Card. The investiga- 


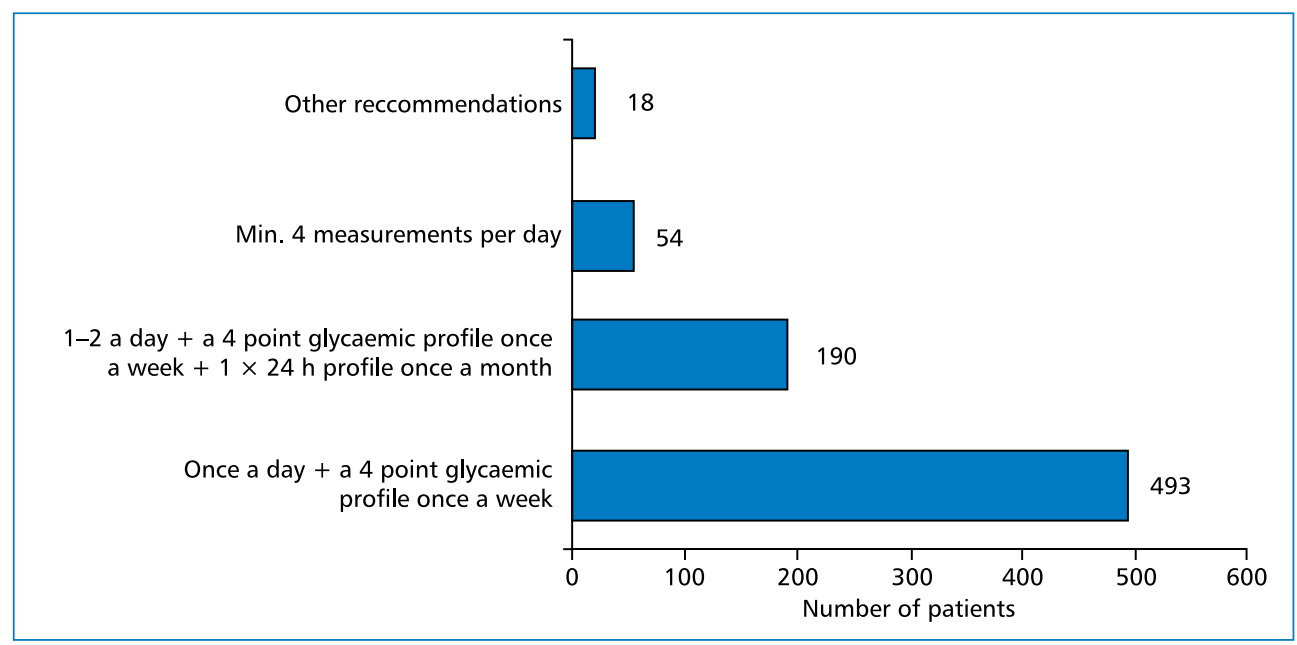

Figure 1. Reccommendations for self monitoring of glycaemia

tors were able to choose from the answers arranged strictly according to the Polish Diabetes Association Recommendations for 2016 (Fig. 1). In 18 cases, the recommendations were marked as "other". These recommendations partly diverged from those defined by the PDA, on the grounds of individual approach to the patient's therapeutic needs and in order to achieve proper glycaemic control. Additionally, the number of test strip boxes prescribed for three months was noted during the first visit.

During the final appointment (V2) set approximately 3 months ( \pm 2 weeks) after the first visit, the investigator assessed the level of compliance to recommendations regarding self monitoring of glycaemia, using the results stored in the memory of the device and the patient's self control diary. He also filled in data on the Patient Observation Card, including the evaluations of glucometer functionalities - the "second chance" measurement among them - made by both the investigator and the patient.

\section{Results}

760 patients were included into the study. Data of 5 patients was excluded from the study due diagnosis of type 1 diabetes. In the studied group 725 patients were diagnosed with type 2 diabetes. The type of diabetes was not determined by the general practitioner in 35 cases. 308 male and 425 female subjects aged accordingly $62.7 \pm 10.7$ and $65.7 \pm 10.9$ were assessed. Of all patients included into the study $685-91.2 \%$ of the group - attended the final appointment. The causes of absence during the final (V2) visit listed by the investigators were inability to contact the patient, isolated cases of withdrawing from the study, losing the glucometer, choosing a different health centre and patients death. Most of these cases were of random nature, while inability to contact the patients was most likely caused by the fact that the date of the second visit was scheduled during the holiday season.

Based on the medical history taken during the first appointment (V1) it was determined that 564 (75.8\%) of patients used oral hypoglycaemic medications and behavioural treatment, 60 patients $(8.1 \%)$ relied solely on behavioural treatment, while 120 patients (16.2\%) used insulin at least once a day. In this last group, insulin therapy was combined with oral hypoglycaemic medications. 51 patients administered insulin once a day; 35 patients used two injections a day and 34 patients performed three injections daily. No information regarding the kind of implemented hypoglycaemic therapy was provided in 16 cases, as well as no explanation of this fact was given.

First of all, the level of type 2 diabetes patients' compliance to recommendations regarding documentation of self monitoring of blood glucose levels - the type of provided data - was assessed. 440 patients (65.1\%) fully complied with the investigators recommendations and brought in both the self control diary and the glucometer to the second appointment. 16 patients $(2.4 \%)$ failed to fulfil the basic, fundamental requirement of providing material regarding their compliance. A similar number of patients provided only one type of material - $14.6 \%$ of participants handed just the control diary, while $17.9 \%$ - the glucometer alone. In 9 cases (1.3\%) no data was obtained regarding self-monitoring in the 3 months \pm 2 weeks.

Secondly, compliance with the Polish Diabetes Association recommendations regarding self-monitoring 
Table 1. Self monitoring assessment - implementation of recommendations for glycaemic control

\begin{tabular}{lcc}
$\begin{array}{l}\text { Was the patient's self-monitoring of glycaemia } \\
\text { in line with the reccommendations? }\end{array}$ & $\mathbf{n}$ & $\%$ \\
\hline Yes & 315 & 46.4 \\
Partly & 326 & 48.0 \\
No & 38 & 5.6 \\
Total & 679 & 100.0 \\
No data & 6 & 0.9 \\
\hline
\end{tabular}

of blood glucose levels was assessed. The results are presented in Table 1. According to the investigators, 315 patients (below 50\%) conscientiously complied with given recommendations. The largest group consisted of patients who partly complied with the recommendations ( $n=326,48.0 \%$ ), while in 38 cases the investigators' suggestions were simply ignored.

In the group of 672 patients who provided data regarding both compliance with recommendations and the type of materials available during V2, a verifying analysis was performed to determine whether those patients - well prepared for the second appointment - also performed better in regard of fulfilment of recommendations for self monitoring of glycaemia, according to the Investigators opinion. Based on the acquired data, it was determined that patients who provided all the received materials during $\mathrm{V} 2$, also exhibited the highest levels of appropriate self monitoring of glycaemia ( $n=276,41.1 \%$ ). The second most numerous group consisted of patients who provided all the required materials, however their self monitoring regimen was only partly in accordance with the physicians suggestions ( $n=157,23.4 \%$ ). The percentages for each level of compliance were similar in the group that provided just the control diary or the glucometer - only $2-3 \%$ of patients fully complied with the recommendations, $11-12 \%$ displayed only partial compliance, while $1.5-2.4 \%$ did not comply at all. Only one patient amongst 16 who did not provide the investigator with any materials followed the recommendations, while the rest showed only partial compliance or no compliance at all.

For a complete picture of compliance with recommendations, the data regarding each specific Investigator was also analyzed. A mean value of answers was determined for each physician, which disclosed the percentage of each investigator's patients fulfilling the recommendations regarding self monitoring. It was assumed that a mean of $100 \%$ indicated that every patient was compliant, while $0 \%$ signalized that no patient at all has fulfilled the recommendations. 16 of 61 Investigators exhibited a high percentage of compliant patients, meaning that 66 to $100 \%$ of their patients fully complied with the recommendations regarding self monitoring of blood glucose. At the same time, 26 investigators demonstrated low percentages of fully compliant patients, indicating that $2 / 3$ of their patients did not perform self monitoring of glycaemia in accordance with the recommendations. For 58 Investigators, the non-compliant part was $1 / 3$ of their patients. Numbers of Investigators whose patients were partly compliant with the guidance, turned out to be spread evenly - 18, 23 and 20 respectively. 29 physicians displayed high effectiveness in motivating the patients towards correct self monitoring performance and returning the received materials - over $2 / 3$ of the patients returned the materials that were previously handed out.

During the DIABCON study information was gathered that allowed verification of the Investigators' compliance to the PDA recommendations regarding self monitoring of blood glucose. It was concluded that for $68,0 \%$ of patients in the public healthcare setting $(n=506)$, arbitrary recommendations of the PDA were implemented. In $1 / 3$ of the subjects, the recommendations regarding self monitoring of blood glucose suggested by the Investigators differed from current guidelines.

In order to assess the Investigators' compliance to guidelines thoroughly, an analysis of individual Investigators was also performed. An average was calculated for each Investigator. It was assumed that if all patients received guidance in accordance with the PDA recommendations, the level of implementation equalled $100 \%$, whereas $0 \%$ indicated that every patient received recommendations diverging from those of the PDA. The results are presented in Table 2 . The results are partly consistent with the data concerning individual patients. Of 62 physicians, 38 suggested self monitoring of glycaemia in line with the PDA recommendations. For 14 physicians, the percentage of patients who received guidance compliant to the guidelines was marked as "medium". For 10 physicians, it was marked as "low".

In the DIABCON study the Investigators also assessed the patients' opinions regarding an additional feature of the Contour Plus ${ }^{\circledR}$ - a "second chance" measurement, which gives the patient an opportunity to complement the required volume of blood to the test strip, if it is insufficient to perform the test. This needs to be done within 30 seconds from the first measurement. $67.7 \%(n=451)$ of patients who answered this question considered the "second chance" measurement useful in everyday self-monitoring. Approximately $30 \%$ 
Table 2. Assessment of implementation of the PDA reccommendations for self-monitoring of blood glucose - analysis of the investigators perspective

\begin{tabular}{lcc}
\hline $\begin{array}{l}\text { Compliance to the PDA self-monitoring } \\
\text { recommendations }\end{array}$ & $\mathbf{n}$ & $\%$ \\
\hline Low $(0-33 \%)$ & 10 & 16.1 \\
Medium (33-66\%) & 14 & 22.6 \\
High (66-100\%) & 38 & 61.3 \\
Total & 62 & 100.0 \\
No data & 0 & 0 \\
\hline
\end{tabular}

( $n=187,28.1 \%$ ) of participants declared that the feature had no importance to them, whereas 28 patients (4.2\%) stated not needing such option at all.

The feature of the Contour Plus ${ }^{\circledR}$ glucometer was also evaluated by the Investigators, regarding correct and effective self monitoring of glycaemia. The physicians pointed out three most important characteristics of the device. Simplicity was chosen 506 times, as the most significant attribute that determines effectiveness of self monitoring of glucose. The second and third most popular choices were capacity memory and accuracy and precision of measurement - 349 and 348 answers respectively. A possibility to measure mean pre- and postprandial glycaemia was also appreciated (Tab. 3). The "second chance" measurement feature and the ability to assess the frequency of glycaemia measurements made by the patient, were marked as the factors least contributing to effective self-monitoring of glycaemia. The assessment of the Contour Plus ${ }^{\circledR}$ glucometer features was in line with the Investigators' opinion regard-
Table 3. "In your opinion, which features or characteristics of the Contour Plus glucometer have the biggest influence on the effectiveness of self-monitoring of glycaemia?" — investigators' responses

\begin{tabular}{lcc}
\hline Investigators' responses & $\mathbf{n}$ & $\%$ \\
\hline Frequency of measurements made by the patient & 176 & 26.1 \\
Accuracy and precision of the glucometer & 348 & 51.6 \\
High capacity memory & 349 & 51.8 \\
Ability to obtain mean glycaemia directly from & 247 & 36.6 \\
the device & & \\
Ability to obtain pre and post prandial glycaemia & 244 & 36.2 \\
directly from the device & & \\
A „second chance” measurement & 178 & 26.4 \\
Simplicity & 506 & 75.1 \\
Total (number of observations) & 674 & 100.0 \\
\hline
\end{tabular}

ing essential data acquired from the device during the control visit, enabling verification of the effectiveness of treatment and its potential modifications (Fig. 2).

The number of test strip boxes prescribed by the Investigators was additional information gathered during the DIABCON study. In order to ascertain if the amount prescribed corresponded with the patients' needs, the number of boxes required to fulfil the recommendations was compared to the actual number prescribed (Tab. 4). Assuming a self monitoring of blood glucose protocol in line with the PDA recommendations, 2 is the minimum number of test strip boxes required by the patient. Among patients who had been recommended to perform 1 blood glucose measurement a day with an additional four-point glycaemic profile once a week,

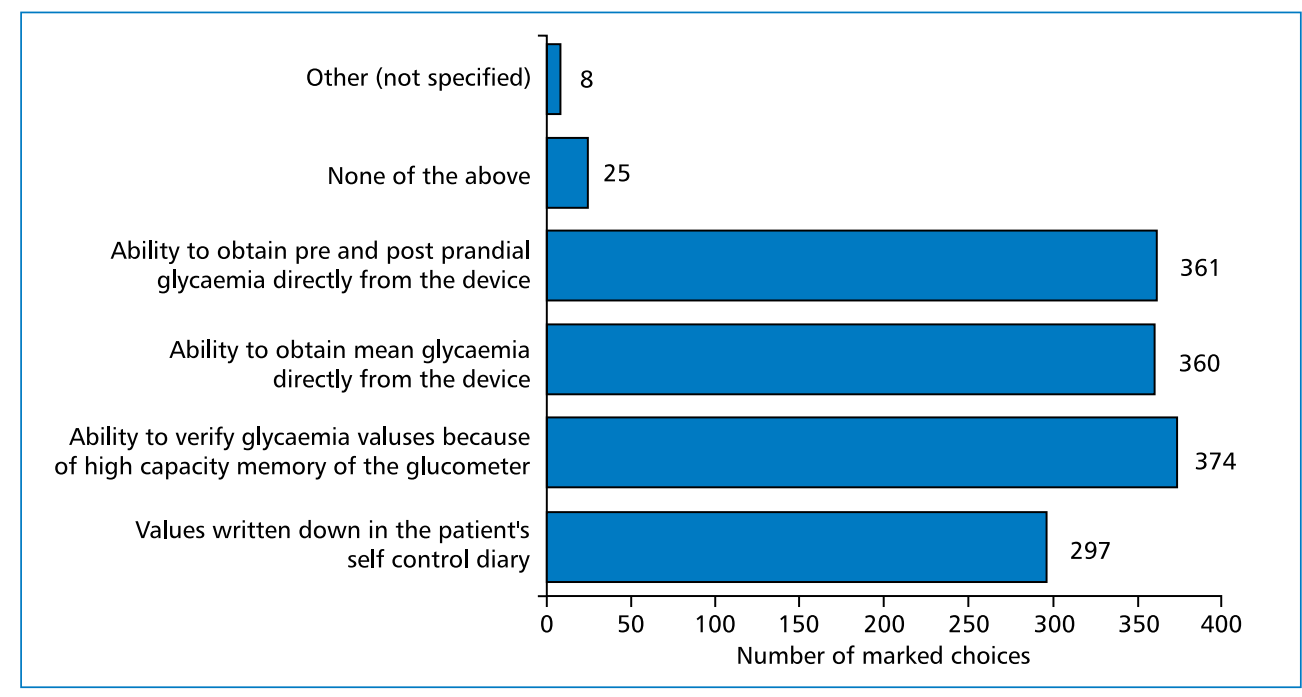

Figure 2. „What data concerning self monitoring of glycaemia are most important to you duirng patients' visits?" - investigators' responses 
Table 4. Number of prescribed test stripn to self control recommendations

\begin{tabular}{|c|c|c|c|}
\hline \multirow[t]{2}{*}{ Recommended number of measurements } & \multicolumn{3}{|c|}{ Number of test strip boxes prescribed } \\
\hline & 1 & 2 & $>2$ \\
\hline $\begin{array}{l}\text { Once a day }+ \text { a } 4 \text { point glycaemic profile once a week } \\
\text { ( } 2 \text { boxes/3 months on average) }\end{array}$ & $58(7.8 \%)$ & $387(51.8 \%)$ & $43(5.8 \%)$ \\
\hline $\begin{array}{l}1-2 \text { a day }+ \text { a } 4 \text { point glycaemic profile once a week }+1 \times 24 \mathrm{~h} \\
\text { profile once a month ( } 4-5 \text { boxes } / 3 \text { months on average) }\end{array}$ & $12(1.6 \%)$ & $82(11.0 \%)$ & $94(12.6 \%)$ \\
\hline Min. 4 a day (5-6 boxes/3 months on average) & $0(0.0 \%)$ & $9(1.2 \%)$ & $45(6.0 \%)$ \\
\hline Other reccommendations & $5(0.7 \%)$ & $6(0.8 \%)$ & $6(0.8 \%)$ \\
\hline Total for each group & $75(10.0 \%)$ & $484(64.8 \%)$ & $188(25.2 \%)$ \\
\hline Total & & 747 (100.0\%) & \\
\hline
\end{tabular}

387 participants had been prescribed a sufficient amount of test strip boxes to perform adequate selfmonitoring, while 43 patients were able to buy even more boxes. Just about a half of patients requiring 4-5 strip boxes every 3 months - 94 participants - was prescribed more than 2 strip boxes. With a demand of 5-6 boxes quarterly, 9 patients were prescribed just 2 boxes, while the numbers of boxes prescribed spread evenly among other recommendation groups. In 161 cases $-21.6 \%$ of the studied population - the number of strips prescribed was insufficient to cover the patient's needs following the self control guidelines. These cases were marked bold in the table.

\section{Discussion}

In the presented report based on the a noninterventional, health education DIABCON programme, a significant discrepancy was observed between the Polish Diabetes Association recommendations for self blood glucose monitoring and its realization among type 2 diabetes patients in the basic healthcare settings. In type 2 diabetes insufficient control often results in incorrect therapeutic decisions, or even serious, life threatening incidents. Thus, the relatively large percentage of non-compliant patients is an important warning sign.

The benefits of correct self-monitoring of glycaemia have been widely discussed in the last decade [5-7]. It was proven that patients with asymptomatic hypoglycaemia and those treated with insulin - especially with the intensive insulin therapy regimen — can benefit from self-monitoring performed with a glucometer [7, 8]. There is some controversy regarding self-monitoring of blood glucose in type 2 diabetes patients treated with oral hypoglycaemic medications. Some research suggests that glycaemia measurements do not influence levels of metabolic control significantly [8]. Perhaps it is a result of either lack of patients' capability to modify the treatment themselves, or no adequate intervention of the physician assessing the data. A number of clinical studies concerning self monitoring of glycaemia have raised the question of data loss, incomplete data or no compliance [7].

In this study, only the implementation of guidelines and the level of metabolic control was assessed, not the correlation between said implementation and levels of metabolic control.

The acquired data shows that, according to the Investigators, patients who were prepared for V2, implemented the recommendations for self monitoring of glycaemia to a greater extent. A conclusion may be drawn, that being given the instruments for glycaemia monitoring is an important motivating factor for the patient, potentially influencing the effectiveness of the treatment. Patients, who retrieved all the materials to the investigator, were more often compliant to given recommendations. Perhaps it was the perspective of the physician having a real, direct ability to verify the given information, that caused the honest control diary management and measurements taking. Participants, who only gave the part of received materials to the Investigator, were also just partly compliant to his recommendations. These results show that an adequate interaction between the physician and the patient, as well as an individual approach to self monitoring of glycaemia, are essential to rising the patient's discipline levels and, as a consequence, acquiring better control of the disease.

The presence of relatively large groups of both Investigators and patients not entirely following the current guidelines, require a deeper look into the sources of such phenomenon. Analysis of recommendations issued by the physicians that were recognized as incompatible with the PDA guidelines, may give rise to suspicions that the difference stems from an individual approach to self monitoring in some patients. However, it cannot be ruled out that general practitioners, who 
take care of patients with different diseases in a basic healthcare setting, do not always follow currently issued guidelines. In both cases, adequate education of not only patients, but also physicians, results in effective self monitoring and, in consequence - better therapy of type 2 diabetes.

Based on the achieved results it may be concluded that effective glucose monitoring is a result of tight cooperation between doctor and patient. Constant evaluation of levels of patients' compliance to given recommendations can be a useful self-assessment tool, improving the care provided to the patient. The fact that $1 / 3$ of the participants retrieved only one form of self monitoring documentation - a glucometer or a diary - may signal the preference distribution regarding traditional or modern way of registering measurements. A preceding conversation in order to assess the patient's preference towards modern or traditional ways of communication may be the key to more effective monitoring of glycaemia.

The opinions on the functionality of the Contour Plus $^{\circledR}$ glucometer were coherent in both the patient and the Investigator groups. Simplicity, high capacity memory, accuracy and precision of measurements were pointed out as clinically significant in both groups. The "second chance" measurement proved to be of little use to the participants in regard to more effective self-monitoring. Perhaps patients, who declared its low utility, perform daily measurements well enough and in accordance to guidelines, so they see no need of additional improvements. On the other hand, a predominant portion of patients positively marked the ability to fill up the amount of tested blood, which suggests that this feature is known to them and used in routine measurements.

Lastly, we should consider the problem of general practitioners prescribing not enough test strips. The reasons for not prescribing test strips that would cover the patients' needs for self monitoring of glycaemia, remains unknown. Perhaps the patients are also under surveillance of a diabetologist, who provides them with enough test strips. However it must not be forgotten that if the patient does not receive a proper tool for self monitoring - in this case, the test strips - the chance of him implementing appropriate self monitoring diminishes, regardless of discipline levels.

\section{Conclusions}

1. A significant discrepancy between Polish Diabetes Association Recommendations of self blood glucose monitoring and its realization by type 2 diabetic patients, carried by general practitioners, was observed.

2. Effective glucose monitoring is dependent on tight cooperation between doctor and patient.

3. Education of both patients and doctors in the matter of current guidelines for self blood glucose monitoring is essential to achieving appropriate and effective self control of diabetes.

\section{Conflict of interest}

The study was conducted in cooperation with Ascensia Diabetes Care Poland.

\section{REFERENCES}

1. Wild S, Roglic G, Green A, et al. Global prevalence of diabetes: estimates for the year 2000 and projections for 2030. Diabetes Care. 2004; 27(5): 1047-1053, doi: 10.2337/diacare.27.5.1047, indexed in Pubmed: 15111519.

2. http://www.who.int/diabetes/en/.

3. Strojek K. Diabetologia Praktyczny Poradnik. Wydanie III. Termedia Wydawnictwo Medyczne, Poznań 2014

4. Gaede $\mathrm{P}$, Lund-Andersen $\mathrm{H}$, Parving $\mathrm{HH}$, et al. Effect of a multifactorial intervention on mortality in type 2 diabetes. N Engl J Med. 2008; 358(6): 580-591, doi: 10.1056/NEJMoa0706245, indexed in Pubmed: 18256393.

5. Schnell O, Alawi H, Battelino T, et al. Self-monitoring of blood glucose in type 2 diabetes: recent studies. J Diabetes Sci Technol. 2013; 7(2): 478-488, doi: 10.1177/193229681300700225, indexed in Pubmed: 23567007.

6. Bergenstal RM, Ahmann AJ, Bailey T, et al. Recommendations for standardizing glucose reporting and analysis to optimize clinical decision making in diabetes: the ambulatory glucose profile. J Diabetes Sci Technol. 2013; 7(2): 562-578, doi: 10.1177/193229681300700234, indexed in Pubmed: 23567014.

7. Schnell O, Barnard K, Bergenstal R, et al. Clinical Utility of SMBG: Recommendations on the Use and Reporting of SMBG in Clinical Research. Diabetes Care. 2015; 38(9): 1627-1633, doi: 10.2337/ /dc14-2919, indexed in Pubmed: 26294772.

8. Nauck MA, Haastert B, Trautner C, et al. Clinical Trials Study Group of the German Association for the Study of Diabetes (Deutsche Diabetes-Gesellschaft). A randomised, controlled trial of selfmonitoring of blood glucose in patients with type 2 diabetes receiving conventional insulin treatment. Diabetologia. 2014; 57(5): 868-877, doi: 10.1007/s00125-014-3168-1, indexed in Pubmed: 24445534. 